\title{
Conductive atomic force microscopy study of InAs growth kinetics on vicinal GaAs (110)
}

\author{
Paloma Tejedor, ${ }^{1, a)}$ Laura Díez-Merino, ${ }^{1}$ Igor Beinik, ${ }^{2}$ and Christian Teichert ${ }^{2}$ \\ ${ }^{1}$ Instituto de Ciencia de Materiales de Madrid, CSIC, Sor Juana Inés de la Cruz 3, 28049 Madrid, Spain \\ ${ }^{2}$ Institut für Physik, Montanuniversität Leoben, Franz. Josef St., 18A-8700 Leoben, Austria
}

(Received 29 May 2009; accepted 13 August 2009; published online 21 September 2009)

\begin{abstract}
Conductive atomic force microscopy has been used to investigate the effect of atomic hydrogen and step orientation on the growth behavior of InAs on GaAs (110) misoriented substrates. Samples grown by conventional molecular beam epitaxy exhibit higher conductivity on [1]0]-multiatomic step edges, where preferential nucleation of InAs nanowires takes place by step decoration. On H-terminated substrates with triangular terraces bounded by [1 $\overline{1} 5]$-type steps, three-dimensional InAs clusters grow selectively at the terrace apices as a result of a kinetically driven enhancement in upward mass transport via $\mathrm{AsH}_{x}$ intermediate species and a reduction in the surface free energy. () 2009 American Institute of Physics. [doi:10.1063/1.3232234]
\end{abstract}

Molecular beam epitaxial (MBE) growth of III-V compounds on (110)-oriented substrates has recently acquired special relevance in the growing field of semiconductor spintronics following the experimental demonstration of a predicted enhancement in the electron spin-relaxation time by more than an order of magnitude in (110)-InAs superlattices relative to the corresponding (001) structures. ${ }^{1-3}$ This improvement in spin dynamics together with the unique characteristics of InAs, which include narrow bandgap, high electron mobility, and strong spin-orbit interaction, make (110) InAs the prime candidate for nonmagnetic, low-power, highspeed spintronic devices, i.e., spin field effect transistors. For high-performance transistors to be exploited in quantum computing or quantum cryptography applications, the ability to grow (110) InAs low-dimensional structures, i.e., nanodots and nanowires, in predefined locations and alignment has to be demonstrated. ${ }^{4,5}$

Self-organized growth on GaAs(110) substrates misoriented toward (111)A is an attractive method to create nanostructured templates ${ }^{6,7}$ for subsequent nucleation of twodimensional (2D) and three-dimensional (3D) arrays of dots and wires having nanoscale dimensions. In previous studies we used a combination of atomic force microscopy (AFM) and in situ reflection high-energy electron diffraction (RHEED) measurements to investigate the variations in growth kinetics and morphology that take place when the (110) vicinal surface is exposed to a beam of atomic hydrogen prior to or during GaAs homoepitaxy from molecular beams of $\mathrm{Ga}$ and $\mathrm{As}_{4}{ }^{8}$ RHEED intensity oscillations, which indicate 2D layer-by-layer growth, were recorded in a much wider range of growth conditions in the presence of chemisorbed $\mathrm{H}$ compared to conventional $\mathrm{MBE}$ growth from $\mathrm{As}_{4}$ or $\mathrm{As}_{2}{ }^{9}{ }^{9}$ The arsenic incorporation coefficient determined from As-induced oscillations during growth on the $\mathrm{H}$-terminated surface was approximately twice that of $\mathrm{As}_{4}$ on the nonexposed surface, i.e., 0.3 at $500{ }^{\circ} \mathrm{C}$, and its value exhibited a temperature independent behavior. In the absence of any molecular beam mass spectrometry data relative to the

\footnotetext{
a) Author to whom correspondence should be addressed. Electronic mail: ptejedor@icmm.csic.es. Tel.: (34) 91 3349088. FAX: (34) 913720623.
}

sticking coefficient of $\mathrm{As}_{4}$ on the (110) surface, these measurements were semiquantitative, but they pointed to a $\mathrm{H}$-mediated enhancement in the As incorporation kinetics. A new reaction pathway was proposed to account for the enhancement in 2D layer-by-layer growth observed by RHEED and the equalization of terrace sizes exhibited by nanostructured templates formed by H-assisted GaAs(110) homoepitaxy, which involved the H-induced lowering of the EhrlichSchwoebel (E-S) kinetic barriers to atomic As incorporation ${ }^{7}$ via dissociation of molecularly adsorbed $\mathrm{AsH}_{x}{ }^{*}$ precursor species, thus favoring the preferential attachment of adatoms to ascending step edges. ${ }^{10}$ The presence of these $\mathrm{AsH}_{x}$ intermediates on the $\mathrm{H}$-terminated $\mathrm{GaAs}(110)$ surface had been previously identified by photoemission yield spectroscopy and high resolution electron energy loss experiments. ${ }^{11}$

Epitaxial growth of InAs on GaAs has been widely investigated for (001)-oriented substrates, where coherent (i.e., dislocation-free) 3D islands, known as quantum dots, form by a modified Stranski-Krastanov growth mode. By contrast, growth of InAs on the GaAs (110) surface follows a layer-by-layer 2D mode irrespective of layer thickness and strain relaxation occurs only by the formation of misfit dislocations. $^{12,13}$ On GaAs (110) substrates misoriented toward (111)A, growth proceeds by a particular type of step flow called step decoration, where nucleation occurs on the surface step edges, forming elongated strips along the main [1] 0$]$-step direction with effectively no nucleation centers on the terraces. ${ }^{14}$ While this growth mode has been exploited to achieve ordered arrays of InAs nanowires selectively nucleated on [1]0]-type bunched steps, ${ }^{15}$ it has been the main obstacle to achieve self-organized InAs quantum dots on vicinal GaAs (110) substrates. In this work we investigate a different approach to modify the growth kinetics of InAs on GaAs (110) substrates misoriented toward (111)A with the aim to induce the spontaneous formation of dotlike nanostructures for potential application to single electron devices. By using conductive AFM (C-AFM) we will show that the combination of the surfactant action of atomic hydrogen with the use of nanostructured buffer templates results in a kinetically driven enhancement in upward mass transport and a reduction in surface free energy, which alters the InAs 

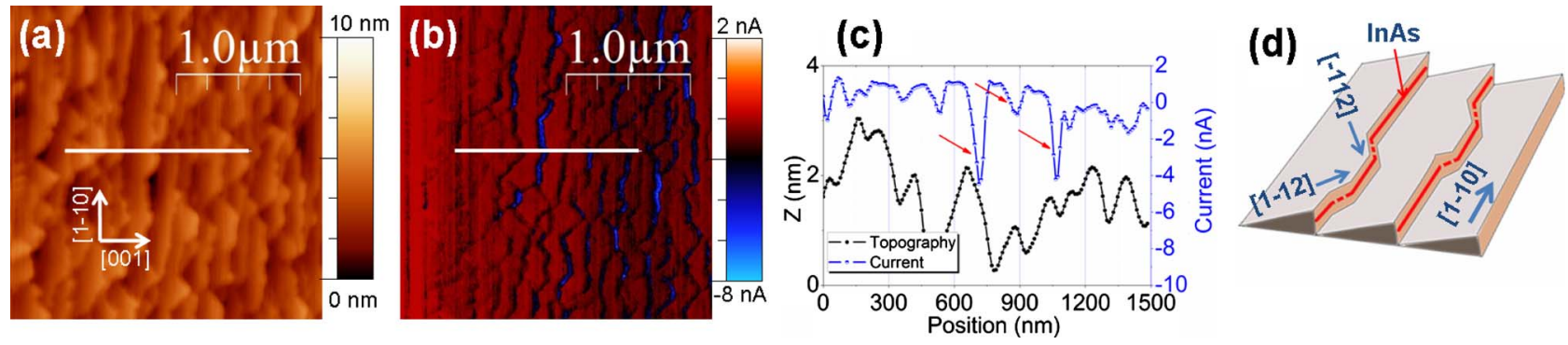

FIG. 1. (Color online) C-AFM (a) topography image in UHV, (b) 2D current map recorded at $-9.1 \mathrm{~V}$ sample bias, (c) corresponding cross-section profiles, and (d) schematic of the ideal terrace topology after deposition of $5 \mathrm{ML}$ InAs on template A. High-current regions indicated by arrows in (c) are coincident with the surface bunched steps.

growth mode and enables the formation of 3D dotlike nanostructures on the GaAs(110) vicinal surface.

The InAs samples were grown in a solid-source Varian MBE-360 system described elsewhere. ${ }^{8}$ The native oxide of the GaAs (110) substrates misoriented toward (111)A by $2^{\circ}$ was removed prior to epitaxial growth under an $\mathrm{As}_{4}$ flux of $(2-3) \times 10^{15}$ molecules $\mathrm{cm}^{-2} \mathrm{~s}^{-1}$ either by thermal desorption at $610^{\circ} \mathrm{C}$ or by atomic $\mathrm{H}$ irradiation at $450{ }^{\circ} \mathrm{C}$. GaAs buffer templates with a thickness of $100 \mathrm{~nm}(500 \mathrm{ML}$ in the [110] direction), hereafter named template $\mathrm{A}$ and template B, were then deposited under different kinetic regimes. Template A was grown under As-rich conditions $\left(430{ }^{\circ} \mathrm{C}\right.$, As: Ga flux ratio of 30) to favor the formation of [110]-oriented bunched steps. The formation of terraces bounded by [1115]-type steps in template $\mathrm{B}$, where the influence of both chemisorbed $\mathrm{H}$ and step orientation on In migration and incorporation kinetics were investigated, was induced during growth under As-deficient conditions $\left(500{ }^{\circ} \mathrm{C}\right.$, As: Ga flux ratio of 10) after $\mathrm{H}$-assisted surface cleaning, as atomic $\mathrm{H}$ promotes the rapid reorganization of the surface steps along $[1 \overline{1} n](n \geq 2)$ in this growth regime. ${ }^{8}$ Subsequently, $5 \mathrm{ML}$ of InAs were deposited on each of these templates under identical As-rich conditions $\left(425{ }^{\circ} \mathrm{C}\right.$, As:In flux ratio of 60) at a growth rate of $0.1 \mathrm{ML} \mathrm{s}^{-1}$. C-AFM measurements were carried out in an ultrahigh vacuum $\mathrm{AFM} /$ scanning tunnelling microscope system (Omicron $\mathrm{GmbH})$ at $3.8 \times 10^{-9}$ torr. The voltage applied to the samples was varied from 0 to $-10 \mathrm{~V}$. A homemade amplifier ${ }^{16}$ allows measurements with overall amplification of order $10^{11}$ and a peak-to-peak noise level of $0.2 \mathrm{pA}$ at a bandwidth of $400 \mathrm{~Hz}$. NT-MDT DCP11 cantilevers with diamond-coated tips $(k=5-12 \mathrm{~N} / \mathrm{m})$ were used as probes. While the macroscopic tip radius of curvature is $\sim 100 \mathrm{~nm}$, the grains of the polycrystalline diamond coating allow a resolution below $20 \mathrm{~nm} .{ }^{17}$

Figure 1 depicts both the topography and current AFM images of the vicinal surface after deposition of 5 ML InAs on template $\mathrm{A}$, together with their corresponding crosssection scan profiles taken along the [001] tilt direction. The image in Fig. 1(a) shows the formation of a characteristic multiatomic step array with step edges running along [1 $1 \overline{1} 0]$, separated by (110)-terraces with an average width of $\sim 160 \mathrm{~nm}$, i.e., the approximate diffusion length of the In adatoms at the temperature of the experiment. The current image in Fig. 1(b) clearly shows stripes of high current running along $[1 \overline{1} 0]$. Comparison of the corresponding crosssection profiles in Fig. 1(c) revealed that the high current regions (bright) coincided with the step bunches running along the $[1 \overline{1} 0]$ direction, thus indicating that InAs grows preferentially on them, forming 3-nm-high and up to 70-nmwide wires of variable length. On the other hand, [1 $\overline{1} 2]$-type steps very rarely appear decorated by InAs. A schematic of the terrace ideal topology is illustrated in Fig. 1(d). The origin of the preferred nucleation of InAs on [1 $1 \overline{1} 0]$ step edges is to be found in its inherent topology. ${ }^{18}$ The dangling bonds of the Ga-terminated $[1 \overline{1} 0]$ step edge cannot rehybridize their charge density to satisfy the autocompensation principle and consequently these steps are very reactive, acting as sinks for diffusing adatoms. In addition, the sticking of adatoms is likely to have a low activation energy because no bonds need to be broken to accommodate the incoming In adatoms. But rehybridization of the dangling bond charge density in the case of $[1 \overline{1} n]$-type steps can be readily achieved by the creation of vacancies and/or step edge rebonding to form As dimers ${ }^{19}$ resulting in very stable step configurations. Therefore, the limiting factor of InAs nucleation on [1 $1 \overline{1} 2]$-type steps at the temperature of our experiments is most probably the high activation energy associated with the insertion of In adatoms into step edge As dimers. This kinetic hindrance induces a current of migrating In adatoms away from $[1 \overline{1} n]$-type steps toward $[1 \overline{1} 0]$-type steps, where incorporation is more favorable.

For comparison, the topography and current AFM images of a 5 ML InAs sample deposited at the same temperature and As:In flux ratio on the H-covered template B, are depicted in Figs. 2(a) and 2(b), respectively. The topography image shows triangular (110) terraces bounded by $[1 \overline{1} 5]$-bunched steps, whose average height is $\sim 10 \mathrm{~nm}$. The chemical contrast achieved in C-AFM images evidences the alteration of the InAs growth mode on the H-covered vicinal GaAs (110) surface. The scan profiles along the tilt direction in Fig. 2(c) show that the highest current areas in this sample are located on the terrace apices where InAs accumulates, forming 3D clusters with an average lateral size of $65 \mathrm{~nm}$. The estimated density of clusters is $\sim 4 \times 10^{9} \mathrm{~cm}^{-2}$. Figure 2(d) illustrates the ideal terrace topology in this sample. Along with the gain in conductivity at the apices, we observe current peaks of lower intensity associated with spatial regions up to $20 \mathrm{~nm}$ in lateral size randomly distributed on the surface terraces, depicted as an inset in Fig. 2(b). It is possible that these conducting regions on the terraces result from a chemical exchange between deposited In adatoms and the substrate surface $\mathrm{Ga}$ atoms. ${ }^{13}$ 

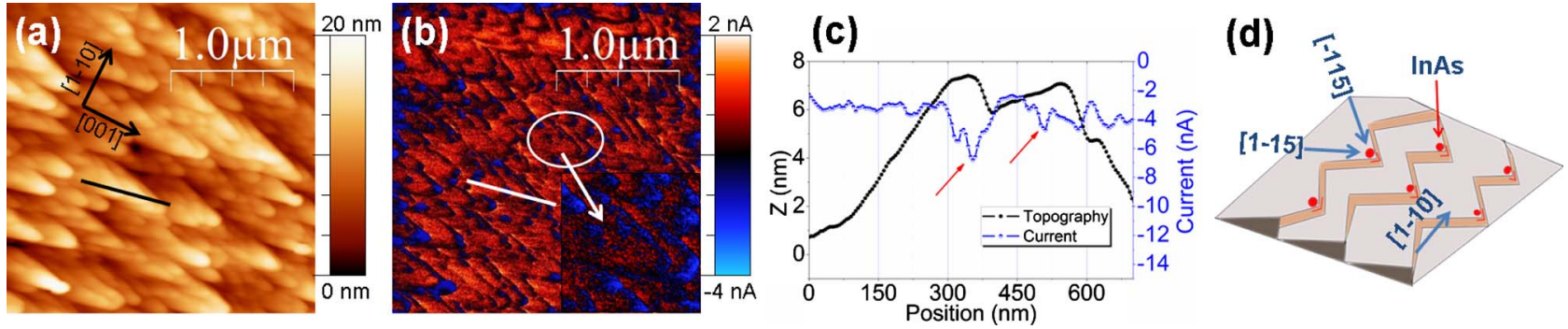

FIG. 2. (Color online) C-AFM (a) topography image in UHV, (b) 2D current map recorded at $-9.1 \mathrm{~V}$ sample bias, (c) corresponding cross-section profiles, and (d) schematic of the ideal terrace topology after deposition of $5 \mathrm{ML}$ of InAs on template B. Coincident areas are marked by arrows in the cross-section profiles. The inset in (b) shows a detail of the discontinuous conducting areas on the terrace.

At the temperature of our experiments, the InAs growth reaction is likely to follow a different pathway on the $\mathrm{H}$-terminated surface, involving $\mathrm{H}$-bonded In adatoms and $\mathrm{AsH}_{x}$ intermediate species. ${ }^{11}$ As shown earlier, H-assisted growth through these intermediate species enhances the As incorporation kinetics, ${ }^{8}$ favoring the preferential attachment of adatoms to upper step and island edges. In our opinion, the most plausible explanation for the observed surface morphology after 5 ML InAs deposition is the destabilization of the growth front due to this preferential attachment of adatoms to ascending step edges in the presence of a weak E-S barrier. According to theoretical models described in Ref. 20, this creates a growth-induced diffusion current in the uphill direction that destabilizes growth on the vicinal surface leading to 3D mound formation, irrespective of the occurrence of an induction layer-by-layer period. In this growth scenario the adatoms arriving at the terraces by upward mass transport or from the deposition flux will tend to incorporate preferentially at the upper side of the steps where InAs material can be partially relaxed, rather than at energetically less favorable sites on the terrace center or at the foot of steps where InAs would be overstrained ${ }^{21}$ in good agreement with Fig. 2(b). Consequently, the selective nucleation at the apices tends to make the protruding parts of the steps unstable toward transverse meandering, ${ }^{22}$ while the strain energy increases until the height of the steps reaches a critical thickness and 3D clusters are formed on the terrace apices. Besides, chemisorbed $\mathrm{H}$ lowers the surface energy of the growing layer favoring the formation of a 3D island morphology. ${ }^{23,24}$ In addition, the height of the clusters can be as high as $50 \AA$, that is, more than three times greater than the nominal $5 \mathrm{ML}$ coverage, which suggests that they have grown by incorporating material both from the incoming flux and from the surrounding layer. In conclusion, the observed change from step decoration to $3 \mathrm{D}$ growth for InAs on the vicinal GaAs (110) surface is consistent with an enhancement in the adatom incorporation kinetics to upper step edges via $\mathrm{AsH}_{x}$ intermediate species and to the reduced free energy of the H-terminated surface. In turn, the specific morphology of the template used in the H-assisted MBE experiment contributes to confine laterally the InAs nanostructures by offering preferred nucleation sites where the transition from $2 \mathrm{D}$ growth to $3 \mathrm{D}$ mound formation is energetically and kinetically most favorable.
This work was supported by the Spanish MEC under Grant Nos. TEC2007-66955 and HU2006-0022, the Austrian Exchange Service (ÖAD) under Grant No. ES 17/2007, and the Austrian Science Fund (FWF) under Grant No. P19636N20.

${ }^{1}$ K. C. Hall, K. Gundogdu, J. L. Hicks, A. N. Kocbay, M. E. Flatté, T. F. Boggess, K. Holabird, A. Hunter, D. H. Chow, and J. J. Zinck, Appl. Phys. Lett. 86, 202114 (2005).

${ }^{2}$ Y. Ohno, R. Terauchi, T. Adachi, F. Matsukura, and H. Ohno, Phys. Rev. Lett. 83, 4196 (1999).

${ }^{3}$ J. T. Olesberg, W. H. Lau, M. E. Flatté, C. Yu, E. Altunkaya, E. M. Shaw, T. C. Hasenberg, and T. F. Boggess, Phys. Rev. B 64, 201301 (2001).

${ }^{4}$ E. Waks, K. Inoue, C. Santori, D. Fatal, J. Vuckovic, G. S. Solomon, and Y. Yamamoto, Nature (London) 420, 762 (2002).

${ }^{5}$ The International Technology Roadmap for Semiconductors, 2008 Update, http://www.itrs.net.

${ }^{6}$ A. Krishnamurthy, M. Lorke, M. Wassermeier, D. R. M. Williams, and P. M. Petroff, J. Vac. Sci. Technol. B 11, 1384 (1993).

${ }^{7}$ P. Tejedor, F. E. Allegretti, P. Šmilauer, and B. A. Joyce, Surf. Sci. 407, 82 (1998)

${ }^{8}$ P. Tejedor, M. L. Crespillo, and B. A. Joyce, Appl. Phys. Lett. 88, 063101 (2006); Mater. Sci. Eng., C 26, 852 (2006).

${ }^{9}$ D. Holmes, J. G. Belk, J. L. Sudijono, J. H. Neave, T. S. Jones, and B. A. Joyce, Appl. Phys. Lett. 67, 2848 (1995).

${ }^{10}$ R. L. Schwoebel and E. J. Shipsey, J. Appl. Phys. 37, 3682 (1966).

${ }^{11}$ S. Nannarone and M. Pedio, Surf. Sci. Rep. 51, 1 (2003).

${ }^{12}$ B. A. Joyce and D. D. Vvedensky, Mater. Sci. Eng. R. 46, 127 (2004), and references therein.

${ }^{13}$ J. G. Belk, D. W. Pashley, C. F. McConville, B. A. Joyce, and T. S. Jones, Surf. Sci. 410, 82 (1998).

${ }^{14}$ X. M. Zhang, D. W. Pashley, I. Kamiya, J. H. Neave, and B. A. Joyce, J. Cryst. Growth 147, 234 (1995).

${ }^{15}$ S. Torii, K. Bando, B.-R. Shim, K. Maehashi, and H. Nakashima, Jpn. J. Appl. Phys., Part 1 38, 4673 (1999).

${ }^{16}$ S. Kremmer, C. Teichert, E. Pischler, H. Gold, F. Kuchar, and M. Schatzmayr, Surf. Interface Anal. 33, 168 (2002).

${ }^{17}$ S. Kremmer, S. Peissl, C. Teichert, F. Kuchar, and H. Hofer, Mater. Sci. Eng., B 102, 88 (2003).

${ }^{18}$ J. M. McCoy and J. P. LaFemina, Phys. Rev. B 54, 14511 (1996).

${ }^{19}$ Y. Liang, W. E. Packard, J. D. Dow, H. Ho, and G. J. Lapeyre, Phys. Rev. B 48, 11942 (1993).

${ }^{20}$ T. Michely and J. Krug, Islands, Mounds and Atoms: Patterns and Processes in Crystal Growth Far from Equilibrium (Springer, Berlin, 2004).

${ }^{21}$ L. Porte, J. Cryst. Growth 273, 136 (2004).

${ }^{22}$ G. S. Bales and A. Zangwill, Phys. Rev. B 55, R1973 (1997).

${ }^{23}$ C. W. Snyder and B. G. Orr, Phys. Rev. Lett. 70, 1030 (1993).

${ }^{24}$ B. Galiana, M. Benedicto, S. Lorbek, G. Hlawacek, C. Teichert, and P. Tejedor (unpublished). 